\title{
ORIGINAL
}

\section{EPIDEMIOLOGÍA DE LA FRACTURA OSTEOPORÓTICA DE CADERA EN LA PROVINCIA DE ZAMORA (1.993)}

\author{
Mariano Izquierdo Sánchez (1), Carlos Ochoa Sangrador (2), Isidoro Sánchez Blanco (1), María \\ del Carmen Hidalgo Prieto (1), Fernando Lozano del Valle (1) y Tomás Martín González (3). \\ (1) Servicio de Rehabilitación. Hospital «Virgen de la Concha». Zamora. \\ (2) Unidad de Investigación. Hospital «Virgen de la Concha». Zamora. \\ (3) Unidad de Endocrinología. Servicio de Medicina Interna. Hospital «Virgen de la Concha». Zamora.
}

\section{RESUMEN}

Fundamentos: Conocer los principales aspectos epidemiologicos de la fractura osteoporótica de cadera (FOC) en la provincia de Zamora en 1993, y las tasas de incidencia acumulada cruda y estandarizada respecto a la población española.

Métodos: Se estudian 215 personas portadoras de FOC en nuestra provincia durante 1993. De ellos, 203 fueron ingresados y, de éstos, 188 se intervinieron quirúrgicamente por parte del servicio de traumatología. Las variables estudiadas fueron: Sexo, edad, ámbito (rural, urbano), tipo de fractura (cervical, trocantérea), procedimiento quirúrgico (prótesis parcial, tallos de Ender, otros), estacionalidad y estancia media hospitalaria.

Resultados: La incidencia acumulada global resultó de $100,6 / 100.000$ habitantes. En los mayores de 50 años la incidencia fue de 246,5/100.000 habitantes (según censo de 1991). Existió un predominio femenino (3,78:1). Las personas con FOC tenían una edad media $81,3 \pm 8,8$ años. Reparto al $50 \%$ del tipo de fractura tanto a nivel global como para el sexo femenino. La incidencia de fractura trocantérea fue el doble el sexo masculino que en el femenino. La estancia media en los pacientes ingresados fue de 15,35 días. La incidencia acumulada estandarizada por edad y sexo para la población española de 1991 sería de 202,99 fracturas por 100.000 habitantes/año, lo que supondría un número total de fracturas de 23,740 fracturas/año.

Conclusiones: La provincia de Zamora tiene una de las mayores incidencias de FOC comunicadas hasta la fecha en España, tanto cruda como específica para mayores de 50 años. La media de edad, el predominio del sexo femenino, la distribución del tipo de fractura y la estancia media son similares a las publicadas en nuestro país.

Palabras clave: Epidemiología. Fractura de cadera. Osteoporosis.

\section{ABSTRACT \\ Epidemiology of Osteoporotic Hip Fracture in the Province of Zamora (1993)}

Background: To identify epidemiological aspects of the osteoporotic hip fractures (FOC) in the province of Zamora in 1993, and the basic and standardised accumulative incidence rates amongst the Spanish population.

Methods: 215 FOC cases were studied in the province throughout 1993. 203 were admitted to hospital, 188 of which were treated surgically by the Traumatology Department. The surveyed variables were: sex, age, location (rural, urban), type of (cervical, trochantereal) fracture, surgical procedure (partial prosthesis, Ender stems, others), seasonal factors and average hospital stay.

Results: Global accumulative incidence: 100.6 per 100,000 inhabitants. With respect to people over 50 , the rate was 246.5 per 100,000 inhabitants (according to the 1991 census). Female predominance (3.78:1). Average age: $81.3 ? 8.8$ years. $50 \%$ distribution of fracture type, both at global and female level. Dual incidence of trochantereal fracture suffered by males. Average stay: 15.35 days. Standardised accumulative incidence according to age and sex for the Spanish population in 1991 would be 202.99 fractures per 100,000 inhabitants and year, which would result in a total number of 23,740 fractures per year.

Conclusions: The province of Zamora currently has onc of the highest rates of FOC in Spain, both with respect to overall population as well as people over 50 years of age. Average age, predominance of the female sex, distribution of the type of frachure and average hospital stay are similar to the figures published in our country.

Key words: Epidemiology. Hip fracture. Osteoporosis.

Correspondencia:

Mariano Izquierdo Sánchez

Calle San Torcuato, $32.2{ }^{\circ} \mathrm{C}$.

Zamora. 


\section{INTRODUCCIÓN}

La fractura osteoporótica de cadera (FOC), está considerada la mayor complicación de la osteoporosis, sobre todo en los países occidentales, y ha sido motivo de estudio desde hace 40 años por los enormes problemas sociales, económicos y sanitarios que plantea. Los estudios epidemiologicos realizados durante estos años, han puesto de manifiesto una prevalencia creciente de la FOC debido, fundamentalmente, al aumento de la longevidad. Las diferencias metodológicas, geográficas y demográficas son muy importantes como ha puesto de manifiesto el estudio MEDOS ${ }^{1}$. Es necesario profundizar en aspectos epidemiologicos de la FOC en los diferentes países y comunidades para un mejor conocimiento de incidencias, factores de riesgo asociados y posibles soluciones ${ }^{2-3}$.

Por otra parte, la asistencia sanitaria a la FOC presenta contrastes muy notables entre los diversos países, comunidades y aún dentro de ellas, habiéndose planteado la necesidad de crear estrategias para seguir prestando asistencia sanitaria adecuada a este colectivo al menor costo económico posible.

En el área sanitaria de Zamora, que cuenta con una población muy envejecida, la patología de la FOC supone una importante proporción del trabajo habitual de los Servicios de Rehabilitación y Traumatología de nuestro Hospital, donde se realiza la practica totalidad de las intervenciones de FOC de la provincia.

Los objetivos del presente trabajo son conocer aspectos epidemiologicos de la FOC en nuestra provincia, fundamentalmente su incidencia por grupos de edad, sexo, ámbito geográfico y tipo de fractura.

\section{PACIENTES Y MÉTODOS}

Se estudian los 215 pacientes, 170 mujeres y 45 hombres, portadores de fractura osteoporólica de cadera que acudieron al Servicio de Urgencias de nuestro Hospital (único Centro de hospitalización traumatológica del Área sanitaria que presta atención quirúrgica a este colectivo) en el periodo comprendido entre el 1 de enero y el 31 de diciembre de 1993. Consideramos fractura osteoporótica de cadera aquella que se produce como consecuencia de un traumatismo menor (habitualmente caída casual) y sin otra causa médica que lo justifique. Se habían excluido, por tanto, los pacientes portadores de fracturas patológicas (tumores primarios o metastásicos) y las fracturas de cadera producidas en grandes traumatismos (accidentes de tráfico, laborales, caídas mayores). Asimismo, se excluyeron a los pacientes menores de 50 años, criterio generalizado en la mayoría de los estudios de incidencia de la FOC en nuestro país, aunque las últimas tendencias sean a excluir los pacientes menores de 65 años. De los 215 pacientes iniciales, 203 fueron ingresados y de éstos, 188 fueron intervenidos por el Servicio de Traumatología.

Para la estimación de las tasas de incidencia de la FOC se han considerado los censos de población de España y de la provincia de Zamora de 1991 del INE (213.668 habitantes) y utilizado como fuente de datos el Libro de Registros del Servicio de Urgencias, las bases de datos de los Servicios de Traumatología y Admisión y las Historias Clínicas del Servicio de Rehabilitación.

Las variables utilizadas han sido las siguientes: Sexo, edad, ámbito (rural: menos de 10.000 habitantes; urbano: mas de 10.000 habitantes), distribución estacional, tipo de fractura (cervical, trocantérea), procedimiento quirúrgico empleado (prótesis total, prótesis parcial, tallos de Ender, osteosíntesis), estacionalidad y estancia media.

Para el análisis estadístico se utilizo la t de Student y ANOVA para la comparación de medias de edades entre grupos. Se calcularon tasas de incidencia acumulada crudas y ajustadas por grupos de sexo, ámbito geo- 
gráfico y tipo de fractura, riesgos relativos para las variables sexo y ámbito geográfico globalmente por tipos de fractura. Los intervalos de confianza se calcularon con el programa Epiinfo (versión 6, módulo EPITABLE).

Asimismo, se realizó estandarización de tasas de incidencia para la población española por método directo.

\section{RESULTADOS}

\section{Características sociodemográficas:}

La provincia de Zamora según el último Censo de población del INE (1991) cuenta con 213.668 habitantes. De ellos, 108.244 son mujeres y 105.424 son hombres. La población mayor de 50 años es de 87.220 habitantes repartidos entre 48.067 mujeres y 39.153 hombres. Con respecto al ámbito, 92.261 habitan en el medio urbano (47.613 mujeres y 44.648 hombres) y 121.407 en el medio rural (60.631 mujeres y 60.776 hombres). La población mayor de 50 años en el ámbito rural estaba compuesta por 59.639 habitantes (32.521 mujeres y 27.118 hombres) y en el ámbito urbano por 27.581 habitantes (15.546 mujeres y 12.035 hombres).

\section{Factores epidemiológicos de la fractura osteoporótica de cadera:}

La incidencia acumulada (IA) cruda de FOC por 100.000 habitantes y año fue de 100,62 , con un intervalo de confianza al 95\% (IC 95\%) de 87-115. La incidencia de FOC para mayores de 50 años por 100.000 habitantes y año fue de 246,5 (IC 95\%: 216 284).

De las 215 fracturas motivo de estudio, 170 correspondieron a mujeres y 45 a hombres (relación mujer/hombre: 3,78/1). Para la población mayor de 50 años y según el sexo, la incidencia fue de 353,67 por 100.000 mujeres y año y de 114,93 por
100.000 hombres y año. El riesgo relativo (RR) del sexo femenino sobre el masculino fue de 3,08 (IC 95\%: 2,22-4,47).

La media de edad global fue de 81,29 años, con una desviación estándar (DE) de 8,82 años. Se observó un incremento exponencial de la FOC con respecto a la edad, sobre todo a partir de los 75 años (Figura 1). La media de edad para la mujer fue de 81,75 años (DE 8,78) y para el hombre de 79,51 años (DE 8,95) $(\mathrm{p}=0,12)$.

La tasa de incidencia acumulada estandarizada esperada para la población española mayor de 50 años (según el Censo Nacional de 1991) fue de 203,99 por 100.000 habitantes/año, siendo para la mujer de 299,30 por 100.000 y para el hombre de 85,49 por 100.000 mayores de 50 años. Para la población española mayor de 50 años se esperarían un total de 23.740 fracturas al año al extrapolar nuestras cifras al Censo Nacional de 1991 (Tabla 1).

De los 215 pacientes inicialmente estudiados, 142 procedían del medio rural $(66 \%)$ y 73 del medio urbano (34\%).

No encontramos diferencias en la media de edad de los pacientes en el medio rural, 81,12 años (DE de 9,5), respecto a los del medio urbano, 81,62 años (DE 7,55) $(\mathrm{p}=0,7)$.

Analizando el sexo de los pacientes de los dos medios se constataba un predominio del sexo femenino más acusado en el medio urbano $(5,1 / 1)$ que en el medio rural $(3,3 / 1)$.

El tipo de fractura pudo ser comprobada en los 188 pacientes intervenidos. De ellos, 92 pacientes $(48,93 \%)$ presentaban fractura cervical y 96 pacientes $(51,07 \%)$ fractura trocantérea. La media de edad para la fractura cervical fue de 79,69 años (DE 8,85 ) y para la fractura trocantérea de 81,45 años (DE 8,84), no encontrándose diferencias significativas entre ambas $(\mathrm{p}=0,17)$. 
Figura 1

Incidencia acumulada de la fractura osteoporótica de cadera en relación con edad y el sexo en la provincia de Zamora

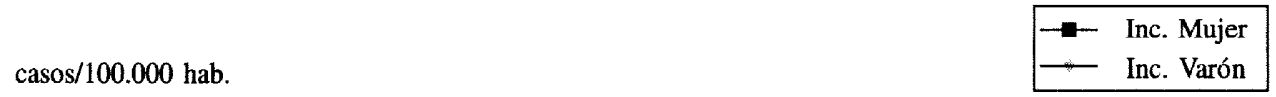

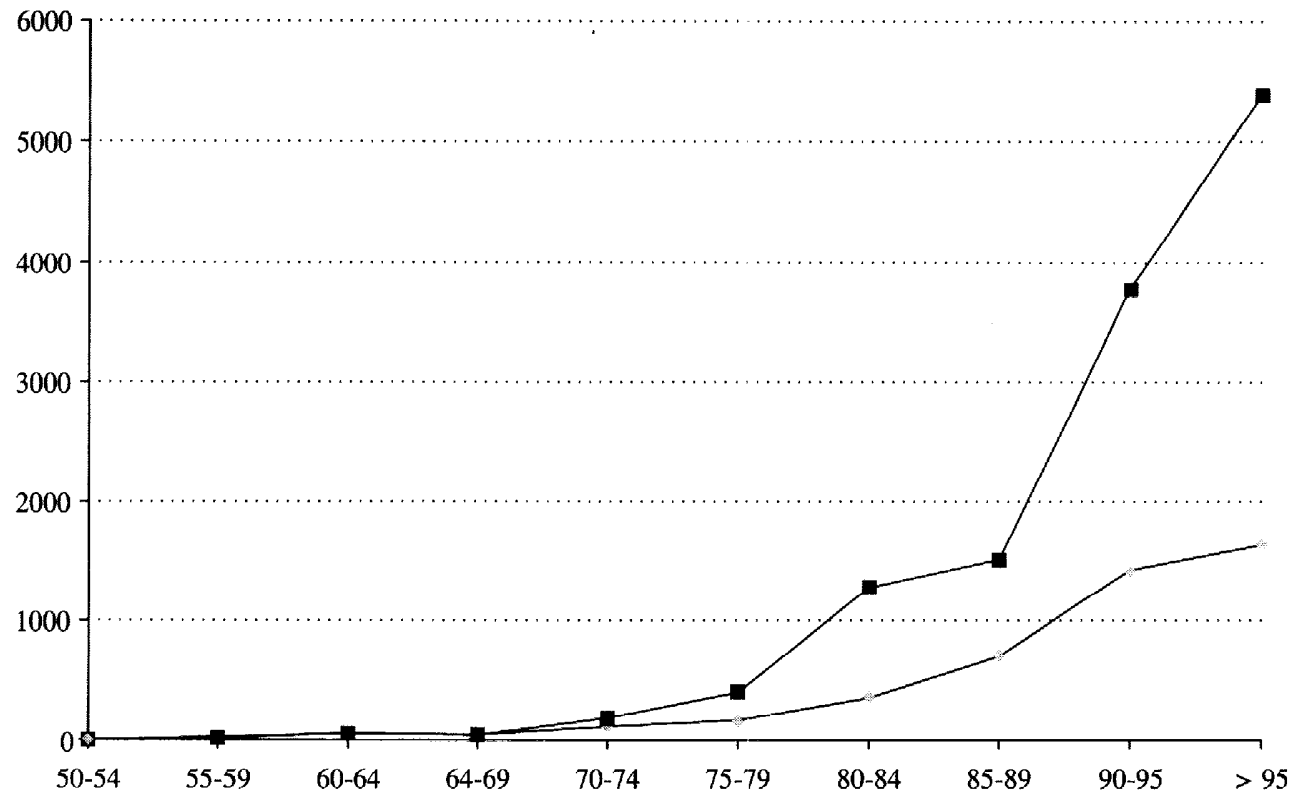

Tabla 1

Frecuencias de fractura osteoporótica de cadera por grupos de edad y sexo para mayores de 50 años. Incidencias acumuladas por 100.000 habitantes, casos esperados para la población nacional (censo 1991) y tasas de incidencia estandarizadas por método directo, globalmente y por sexos

\begin{tabular}{|c|c|c|c|c|c|c|c|c|}
\hline \multirow[b]{2}{*}{$\begin{array}{c}\text { Edad } \\
(\text { años })\end{array}$} & \multicolumn{4}{|c|}{ MUJERES } & \multicolumn{4}{|c|}{ HOMBRES } \\
\hline & $\begin{array}{c}N .^{\circ} \\
\text { casos }\end{array}$ & $\begin{array}{l}\text { IA/I00.000 } \\
\text { Zamora }\end{array}$ & $\begin{array}{l}\text { Habitantes } \\
\text { España }\end{array}$ & $\begin{array}{l}N .^{\circ} \text { de fracturas } \\
\text { esperadas }\end{array}$ & $\begin{array}{l}N .^{\circ} \\
\text { casos }\end{array}$ & $\begin{array}{l}\text { IA/I00.000 } \\
\text { Zamora }\end{array}$ & $\begin{array}{l}\text { Habitantes } \\
\text { España }\end{array}$ & $\begin{array}{l}N .^{\circ} \text { de facturas } \\
\text { esperadas }\end{array}$ \\
\hline $50-54$ & 1 & 16,62 & 1.008 .239 & 167,57 & 0 & 0 & 964.961 & 0 \\
\hline $55-59$ & 2 & 25,95 & 1.153 .216 & 299,26 & 0 & 0 & 1.086 .317 & 0 \\
\hline $60-64$ & 6 & 68,83 & 1.105 .315 & 760,79 & 4 & 53,89 & 1.002 .129 & 540,05 \\
\hline $65-69$ & 4 & 52,21 & 989.769 & 516,76 & 3 & 42,71 & 844.266 & 360,59 \\
\hline $70-74$ & 18 & 295,80 & 774.254 & $2.290,24$ & 6 & 124,76 & 561.392 & 700,39 \\
\hline $75-79$ & 24 & 414,86 & 641.737 & $2.662,31$ & 7 & 178,48 & 410.966 & 733,49 \\
\hline $80-84$ & 50 & $1.279,75$ & 445.807 & $5.705,22$ & 10 & 368,73 & 252.288 & 930,26 \\
\hline $85-89$ & 32 & $1.510,14$ & 226.134 & $3.414,94$ & 9 & 712,58 & 107.529 & 766,23 \\
\hline $90-94$ & 24 & $3.773,58$ & 69.285 & $2.614,52$ & 5 & $1.412,42$ & 26.941 & 380,52 \\
\hline$>95$ & 9 & $5.389,22$ & 15.029 & 809,95 & 1 & $1.639,34$ & 5.324 & 87,28 \\
\hline TOTAL & 170 & & 6.428 .785 & $19.241,55$ & 45 & & 5.262 .113 & $4.498,08$ \\
\hline \multicolumn{5}{|c|}{$\begin{array}{l}\text { INCIDENCIA NACIONAL ESPERADA/100.000 } \\
\text { MUJERES: } 299,30\end{array}$} & \multicolumn{4}{|c|}{$\begin{array}{c}\text { INCIDENCIA NACIONAL ESPERADA/100.000 } \\
\text { HOMBRES: } 85,49\end{array}$} \\
\hline \multicolumn{9}{|c|}{ INCIDENCIA NACIONAL ESTIMADA Y AJUSTADA/100.000 HABITANTES: 202,99} \\
\hline
\end{tabular}

FOC: Fractura osteoporotica de cadera. IA: Incidencia acumulada. 
Figura 2

Incidencia de la fractura osteoporótica de cadera en diferentes provincias españolas para la población mayor de 50 años por sexos

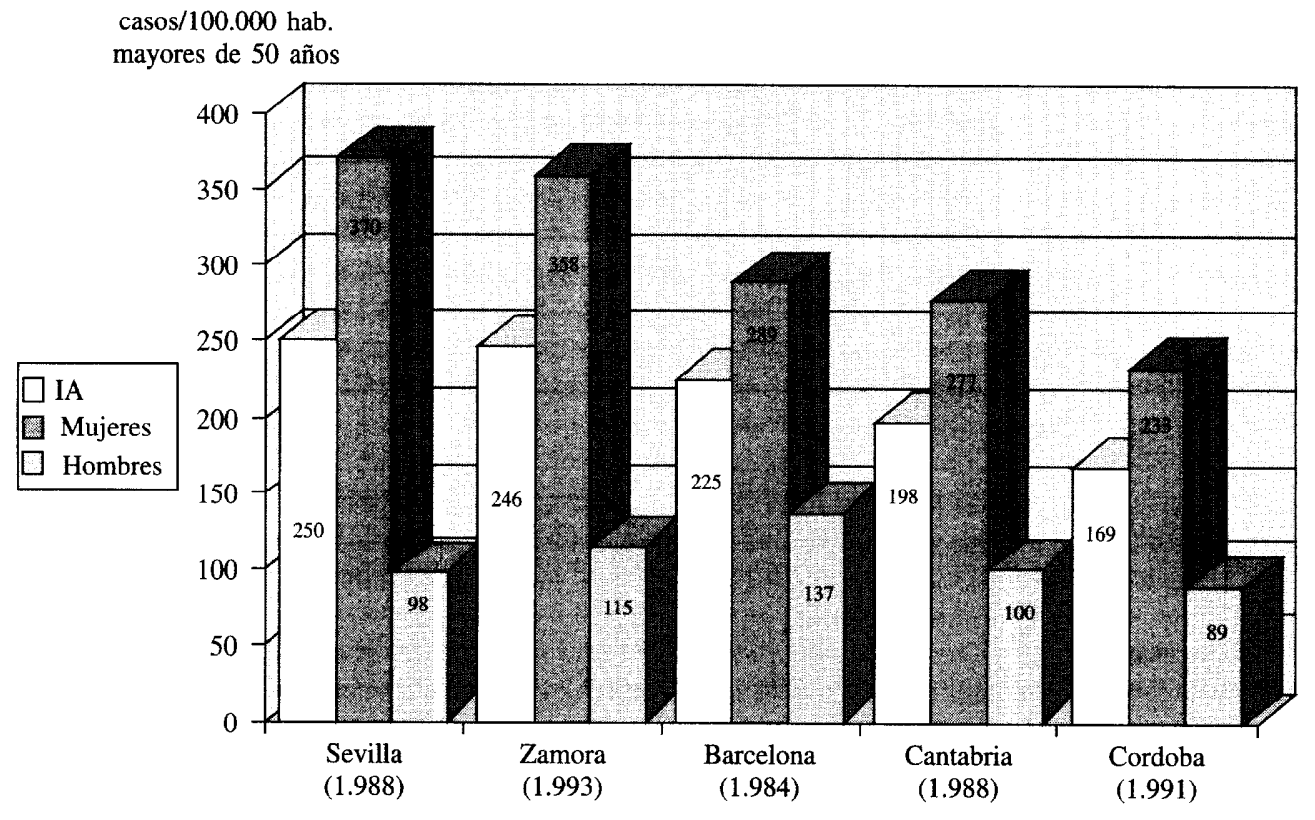

En la tabla 2 se presenta la distribución de las fracturas según sexo y medio para los mayores de 50 años, su IA con sus IC y los RR correspondientes. Asimismo se relaciona la variable tipo de fractura con las variables sexo y ámbito también para la población mayor de 50 años con sus riesgos relativos e intervalos de confianza correspondientes.

\section{Factores hospitalarios:}

De los 215 pacientes iniciales, 203 fueron ingresados $(94,41 \%)$ y de ellos, 188 $(92,61 \%)$ fueron intervenidos por el Servicio de Traumatología. Los 188 pacientes ingresados e intervenidos se distribuyeron entre 153 mujeres $(81,4 \%)$ y 35 hombres $(18,6 \%)$.

La media de edad de los 12 pacientes que no ingresaron fue de 85,55 años (DE 7,78) y en los 188 pacientes intervenidos fue de 80,59 años (DE 8,86) $(\mathrm{p}<0,01)$.

La FOC en nuestra provincia fue mas frecuente en invierno y verano que en otoño y primavera, aunque sin obtenerse diferencias significativas entre ellas (Figura 3). Tampoco se obtuvieron diferencias significativas cuando se relacionó el tipo de fractura, sexo o ámbito con la estacionalidad.

La distribución de los pacientes según el procedimiento quirúrgico empleado fue la siguiente: 90 pacientes $(47,87 \%$ del total $)$ fueron intervenidos mediante prótesis parcial, 92 pacientes $(48,93 \%)$ mediante tallos de Ender y solo 6 pacientes $(3,19 \%)$ con otro tipo de material de osteosíntesis. De los 90 pacientes intervenidos mediante prótesis parcial, 78 eran mujeres $(86,7 \%)$ y 12 eran hombres $(13,3 \%)$. Los pacientes intervenidos mediante Tallos de Ender se distribuyeron entre 71 mujeres $(77,2 \%)$ y 21 hombres 
Tabla 2

Frecuencias e Incidencias Acumuladas de fractura osteoporótica de cadera por sexos y ámbito geográfico, globalmente y por tipos de fractura para la población mayor de 50 años. Riesgos relativos con intervalos de confianza del sexo femenino respecto al masculino y del medio urbano respecto del rural. Riesgo relativo e intervalo de confianza del medio urbano respecto del rural ajustado a sexo

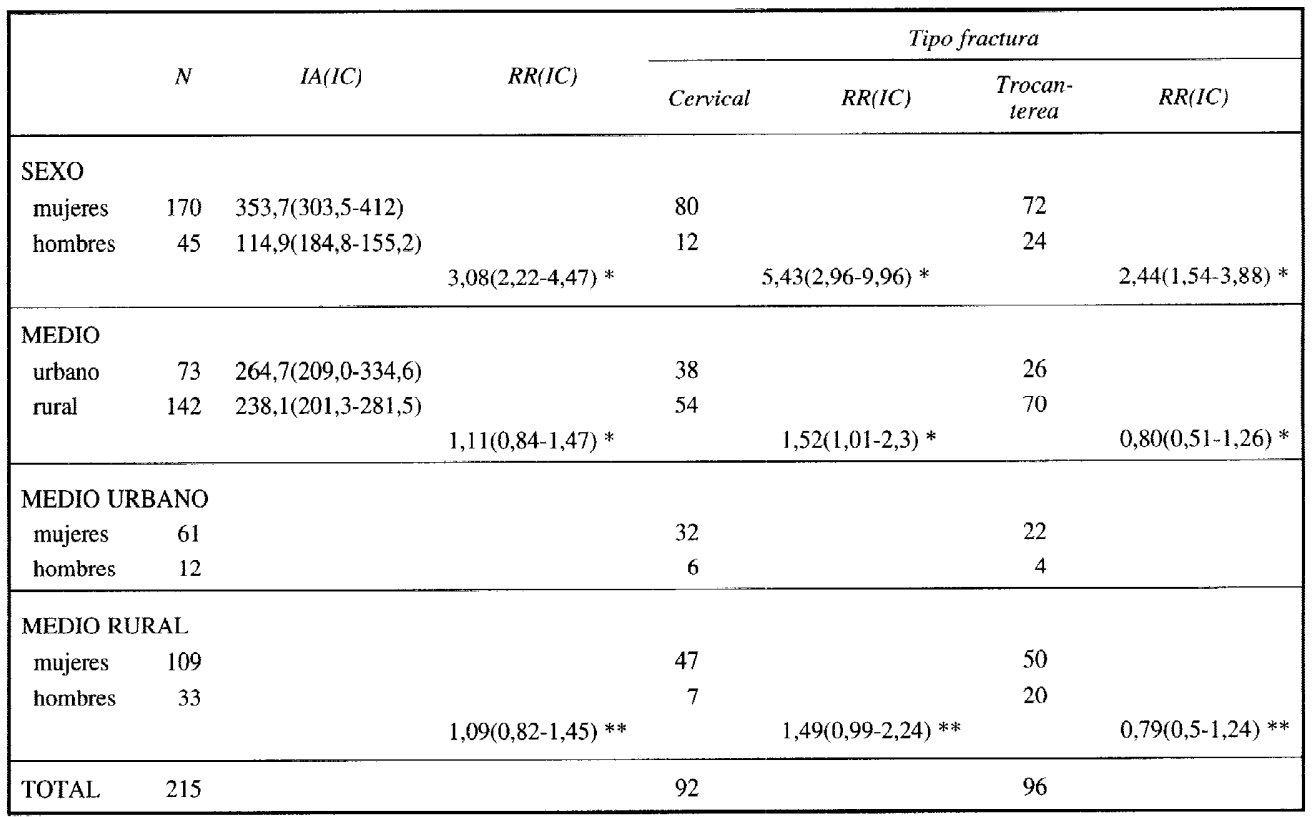

FOC: Fractura osteoporótica de cadera. IA: Incidencia acumulada. * Riesgo relativo crudo. ** Riesgo relativo ajustado por sexo. RR(IC); Riesgo relativo (Intervalo de confianza al $95 \%$ ).

$(22,8 \%)$. Las 6 osteosíntesis intervenidas correspondieron a 4 mujeres $(66,66 \%)$ y 2 hombres $(33,33 \%)$.

Los pacientes intervenidos mediante prótesis parcial tenían una media de edad de 79,95 años (DE 8,73), los pacientes con tallos de Ender de 82,34 años (DE 7,78) y los pacientes con otro tipo de osteosíntesis de 63,16 años (DE 7,33). (p<0,01).

La estancia media global de los 203 pacientes ingresados fue de 15,2 días (DE $7,38)$. La estancia media de los 188 pacientes intervenidos fue de 15,35 días (DE 7,37) y la estancia media de los 15 pacientes ingresados no intervenidos fue de y de 13,47 días (DE 7,88) $(\mathrm{p}=0,34)$. La mediana obtenida fue de 15 días, no encontrándose dife- rencias significativas con respecto a los distintos tipos quirúrgicos.

\section{DISCUSIÓN}

La provincia de Zamora presenta características demográficas y sanitarias de gran interés epidemiológico para el estudio de la FOC. El 40,82\% de la población tiene mas de 50 años y el $22,9 \%$ tiene mas de 65 años. La despoblación sufrida entre 1950 y 1991 supuso una perdida para nuestra provincia del 32,49\% de la población. Esta circunstancia condiciona una alta incidencia de la FOC con importantes repercusiones sanitarias y socioeconómicas, dado que esta patología se encuentra íntimamente ligada al factor edad. Otro hecho a tener en cuenta en el presente estudio es el predominio de la población ru- 
Figura 3

Distribución estacional de la fractura osteoporótica de cadera en Zamora

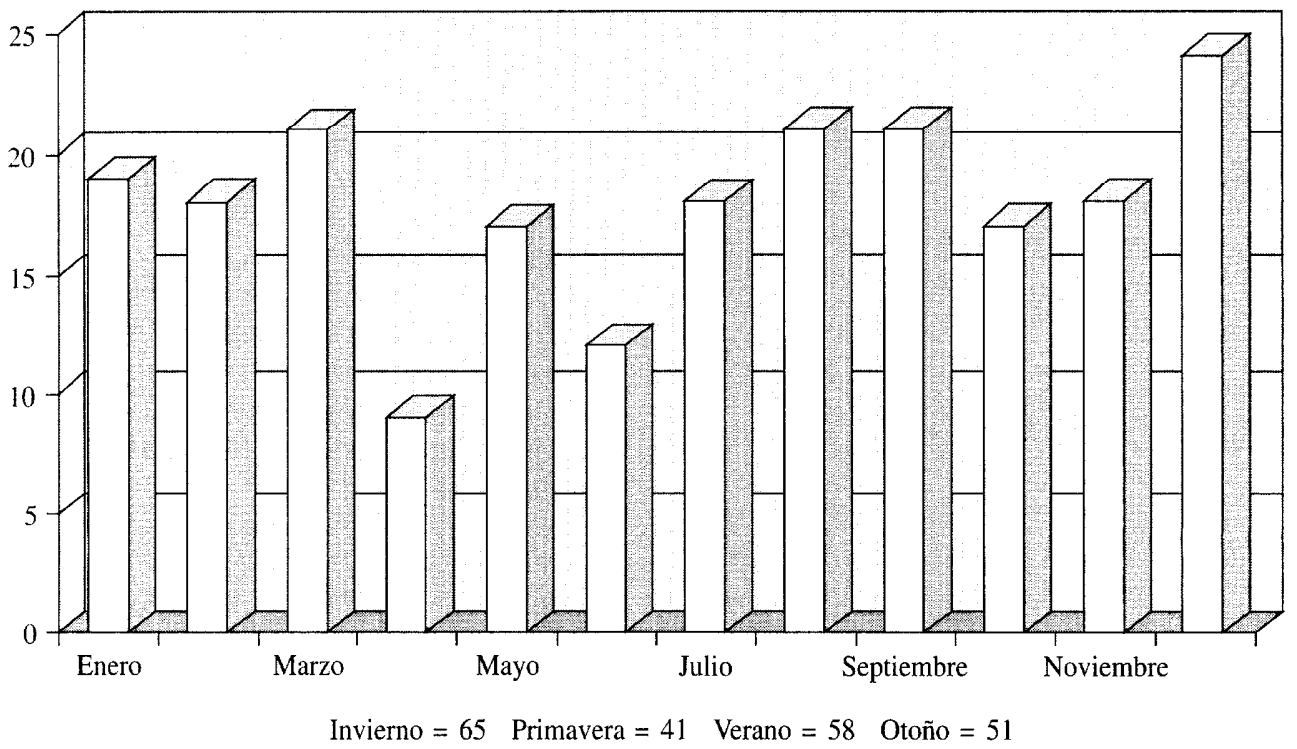

ral $(56,82 \%$ del total) y el que tan sólo dos poblaciones y la capital superen los 10.000 habitantes. Así mismo, dicho estudio se ve favorecido al pertenecer toda la provincia a una sola Área sanitaria con un único Hospital público de referencia.

Las diferencias metodológicas entre estimaciones de incidencia de la fractura osteoporótica de cadera y las variaciones que se producen entre las comunidades geográficas son hechos constatables en toda la literatura consultada ${ }^{1-4}$. Como ya ha sido señalado en nuestro pais ${ }^{4}$, la falta de consenso para establecer la edad en que debe de considerarse fractura osteoporótica o para establecer cifras de incidencia ajustadas a edad y sexo, unido a las discrepancias que se producen entre los diferentes años en que se realizan los estudios, hacen difícil el estudio comparativo de la FOC. Hemos intentado, en el presente estudio, atenernos a los consensos más comunes utilizados en el estudio de la FOC. La incidencia global de nuestro estudio resultó ser de 100,62/100.000 habitantes, cifra considerablemente mayor a la publicada tanto a nivel nacional ${ }^{3-9}$ como en muchos países del sur de Europa ${ }^{1-3}$. En nuestro país, solo los estudios publicados de Sevilla en 1988 (83/100.000 habitantes) y de Barcelona en 1989 (82/100.000 habitantes) se le acercan ${ }^{4}$. Para la población mayor de 50 años, rango que parece ser el mas adecuado para el estudio de la fractura de cade$\mathrm{ra}^{1,3,4,11}$, la incidencia cruda resultó ser de 246,5/100.000 habitantes, cifra ligeramente inferior a la obtenida en la ciudad de Sevilla dentro del estudio $\mathrm{MEDOS}^{3}$, en el que no se llega a concretar la población estudiada ni cruda ni por sexos, y superior en mayor o menor cuantía a las comunicadas en otros puntos de la geografía nacional ${ }^{4,6,8,12}$.

La tasa de IA para las mujeres mayores de 50 años por 100.000 habitantes fue de 353,67 y de 114,93 para los hombres, siguiendo prácticamente el mismo comportamiento de la IA cruda (figura 2): Inferior a 
la obtenida en Sevilla ${ }^{3}$ (370 y 98 respectivamente por 100.000 habitantes) y superior a las referidas a Barcelona ${ }^{13}$, Cantabria ${ }^{9}$, Cordoba $^{11}$, Salamanca ${ }^{7}$ o Madrid ${ }^{12}$. En el estudio de Barcelona, el cálculo de IA cruda se hizo en base a correcciones sobre pérdidas que pudieran producirse. Las tasas de IA crudas para los estudios de Cantabria ${ }^{9}$ y Córdoba ${ }^{11}$ se hicieron para la población mayor de 49 años. La tasa de IA cruda para el estudio de Asturias ${ }^{6}$ no pudo calcularse al no disponer de la población por sexos mayor de 50 años, si bien su IC está dentro del nuestro, por lo que podemos deducir que sus tasas de IA sean similares a las nuestras.

Nuestra incidencia es similar a la encontrada en La Plata (Argentina), al presentar parecidas características de población, número de fracturas y distribución ${ }^{14}$. Cifras sensiblemente mayores son las publicadas en Nueva Zelanda ${ }^{15}$. En estudios europeos únicamente los países nórdicos y centroeuropeos han publicado incidencias superio$\operatorname{res}^{1,2,5,16,17}$. Muy superiores son las referidas a EE.UU. (300 casos nuevos/100.000 habitantes). mayores de 49 años), y sobre todo Noruega (cerca de 1.000) y Suecia ${ }^{\mathrm{l}-3}$.

Se comprueba en éste estudio, como en otros anteriores, un aumento exponencial del numero de fracturas en relación con la edad, sobre todo a partir de los 75 años y para ambos sexos $1,2,5,6,9,10,13$, sólo frenada en el tramo 65-70 años ${ }^{6}$. Dicha tendencia es similar a la publicada en estudios america$\operatorname{nos}^{1,2}$. Quizá ello sea debido a las características propias de población y distribución demográfica de nuestra provincia. En nuestro caso y como señalan diversos autores ${ }^{1-3,6}$ la edad es el principal factor «precipitante» de la fractura osteoporótica de cadera y no otros «agentes» como la latitud geográfica, la climatología o el exceso del estado de bienestar ${ }^{5}$.

La media de edad de nuestros pacientes, 81,29 años, es algo superior o similar a la reflejada en otros estudios nacionales $^{4,6-9,11-12}$. Como en la mayoría de los trabajos publicados ${ }^{6-8,10-11}$ encontramos una mayor edad para las mujeres que para los hombres. En relación al hábitat, la media de edad es superior en el medio urbano sobre el rural, al igual que lo ya referido a nivel nacional $^{6}$.

Se constata, como en otros trabajos ${ }^{6,7,11}$, una mayor edad para los portadores de fractura trocantérea que para los portadores de fractura cervical.

El predominio del sexo femenino ampliamente refrendado en toda la literatura ${ }^{1,3,6-18}$ se confirma en éste estudio. En nuestro caso este predominio femenino es superior al publicado a nivel nacional ${ }^{4}$, con la excepción de Sevilla $(4,7 / 1)$ y Oviedo $(3,8 / 1)$, aunque cuando se tiene en cuenta las poblaciones de ambos sexos y para la población mayor de 50 años, este predominio baja al 3,08/1.

En relación con la tasa de $1 \mathrm{~A}$ por grupos de edad y sexo, los únicos referentes encontrados en la bibliografía a nivel nacional de similares características al nuestro, son los realizados en Sevilla y Madrid $^{3}$ (dentro del estudio MEDOS) y el estudio realizado en Barcelona en $1984^{13}$, encontrándose discrepancias tanto en la diferente incidencia como en la distribución de sexos. Ello puede explicarse tanto por la diferencia de tiempo en que se realizaron dichos estudios como en la diferente distribución de las pirámides de población.

En la bibliografía consultada no hemos encontrado referente alguno de tasas estandarizadas por edad y sexo respecto a la población española. Los cálculos realizados hasta la fecha ${ }^{20}$ se han limitado a calcular el número de fracturas esperadas extrapolando el número global de fracturas según la población de su estudio a todo el territorio nacional de 1984, con la lógica discrepancia respecto al nuestro, fundamentalmente por tratarse de poblaciones nacionales diferentes en diferentes años, no siendo comparables con nuestros resultados.

En lo referido a la topografía de la fractura, en el presente estudio hay un reparto casi 
al $50 \%$ entre fracturas cervicales y trocantéreas. Este equilibrio también se constató en otros trabajos nacionales ${ }^{6,7}$, exceptuando un estudio ${ }^{11}$ en el que se apreció un predominio de la fractura trocantérea.

Cuando estudiamos la relación entre la FOC y la estacionalidad observamos, como en otros trabajos publicados en nuestro pais ${ }^{8,11}$, una mayor incidencia en la estación invernal, en contraste con lo que ocurre en Salamanca ${ }^{7}$ o Asturias ${ }^{6}$, aunque no observamos diferencias significativas entre las diferentes estaciones. Respecto al ámbito geográfico, no encontramos una mayor incidencia en un medio que en otro, pues si bien es cierto que hay un mayor número de fracturas en el medio rural que en el urbano, también es verdad que hay mayor número de habitantes mayores de 50 años en dicho medio. Este equilibrio con respecto al ámbito, también ocurre en otros puntos de nuestra geografía (Cantabria ${ }^{8}$. Las Palmas ${ }^{10}$ ), en contraste con otros (Cordoba ${ }^{11}$, Asturias ${ }^{6}$ ) donde la incidencia de fracturas fue discretamente superior en el ámbito urbano. El mismo equilibrio se observa también fuera de nuestras fronteras en Rochester ${ }^{21}$ y Oslo ${ }^{22}$.

Cuando se relaciona la topografía de la fractura y el sexo para la población mayor de 50 años, comprobamos un predominio mas acusado del sexo femenino sobre el masculino para la fractura cervical que para la fractura trocantérea. Cuando relacionamos tipo de fractura y ámbito geográfico para el mismo tipo de pacientes, se observó que había un riesgo relativo crudo significativo de padecer una fractura cervical si se pertenecía al medio urbano. No se encontraron diferencias significativas cuando se relaciono tipo de fractura y ámbito si se ajustó por sexos en pacientes mayores de 50 años, aunque, casi llega a serlo la pertenencia al medio urbano para padecer una fractura cervical, en contraste con lo que ocurre en Córdoba ${ }^{11}$, done no encuentran diferencias para las fracturas cervicales pero sí hay mayor incidencia de fractura trocantérea en el medio rural.

En relación a los aspectos hospitalarios, la mayor parte de los pacientes con FOC son ingresados e intervenidos, al igual que ocurre en otros trabajos publicados ${ }^{1,4,6-8,10-12}$. Comprobamos un alto índice de ingresos, $94,41 \%$, de los que la mayoría son intervenidos, cifras similares a las obtenidas en otros estudios ${ }^{1.4 .6 .8 .10-12}$, aunque en algunos casos solo se evaluaron los pacientes ingre$\operatorname{sados}^{7-9,13}$. La media de edad de los pacientes que ingresan es sensiblemente inferior a los no ingresados, extremo que no ha podido ser contrastado en la literatura.

La estancia media de los pacientes intervenidos es de 15,35 días, similar a las obtenidas en Valladolid ${ }^{9}$, Córdoba ${ }^{11}$, Cantabria ${ }^{9}$, netamente inferiores a las obtenidas en Barcelona, Madrid, Sevilla, Oviedo ${ }^{4}$ y muy inferiores a las publicadas en Europa y Estados Unidos $^{17,22-26}$. Los estudios realizados al respecto comprenden diversos Sistemas de Salud y aun dentro de un mismo país se constatan diferencias apreciables, en función de la disposición o no de amas por parte de los Servicios de Rehabilitación. La estancia media de los pacientes ingresados y no intervenidos aun siendo menor no demuestra diferencias significativas con respecto a la estancia media global. Se trata de un pequeño colectivo pero heterogéneo que incluye pacientes con fracturas subsidiarias de tratamiento ortopédico (baja estancia) y a pacientes de mal pronóstico con morbimortalidad alta (estancia dispar).

Nuestro hospital no dispone de dichas camas en el servicio de rehabilitación, como tampoco disponemos de un hospital de media-larga estancia, con lo que la mayor parte de los pacientes vuelven a su domicilio habitual. Lo mismo ocurre en otros lugares de la geografía nacional ${ }^{6}$. Este hecho condiciona en gran medida el futuro de muchos de nuestros pacientes, provocando una importante sobrecarga familiar, tal y como han puesto de manifiesto diferentes autores ${ }^{5,9}$. 
Por esta razón el adiestramiento a la fami$\mathrm{lia}^{28-30}$ en el periodo de internamiento se hace imprescindible. En nuestro caso éste aspecto se ve aún más agudizado por el hecho de que son los cónyuges los responsables de hacerlo, presentando características de edad y morbilidad similares a los propios pacientes. Así, ha sido preciso implantar en nuestro centro un programa de rehabilitación ambulatoria para seguir prestando atención rehabilitadora a un grupo seleccionado de pacientes. Este extremo ha sido señalado por diversos autore ${ }^{27-31}$ como un hecho más dentro de estrategias sanitarias ante un problema sanitario y económico de gran trascendencia en el momento actual.

En la actualidad están siendo estudiados los beneficios de tratamientos con vitamina $\mathrm{D}^{32}$, calcio, bifosfonatos y calcitonina, que, junto con a otro tipo de medidas preventivas como el ejercicio físico o el tratamiento de enfermedades concomitantes, puedan disminuir la incidencia de esta enfermedad.

Si bien es cierto que en los últimos años se ha producido un aumento considerable de estudios sobre la FOC, creemos se debe insistir en profundizar en los mismos, incluyendo todos los aspectos, tanto sociales como sanitarios, dada la enorme trascendencia que para nuestro país implica el tratamiento y repercusiones de la FOC en los umbrales del siglo XXI.

Es nuestro propósito analizar en estudios posteriores otra serie de variables fundamentales en el estudio de la FOC tales como estado mental previo, autonomía de la marcha previa a la caída, morbilidad y mortalidad, entre otros, con el fin de conocer mas en profundidad el comportamiento de la FOC en nuestra provincia.

\section{BIBLIOGRAFÍA}

1. Kanis J.A. Epidemiología de la fractura de cadera en Europa: El estudio MEDOS. Rev Clín Esp 1991; 188 Supl 2: 16-19.
2. Johnell O, Gullberg B, Allander E, Kanis and the MEDOS Study Group. The apparent Incidence of Hip Fracture in Europe: A Study of National Register Sources. Osteopor Int 1992; 2: 298-302.

3. Elffors $Y$, Allander E, Kanis J A, Gullberg B, Johnell $O$, Dequeker $J$, et al). The variable incidence of hip fracture in South Europe: The MEDOS Study. Osteopor Int 1994; 4: 253-263.

4. Sosa Henriquez M. La fractura osteoporótica de cadera en España. Rev Esp Enf Metab Oseas 1993; 2: $189-192$

5. Navarro Quilis A. Fractura osteoporótica de fémur en España. Med Clín (Barc) 1989; 92: 733-735.

6. Altalill Aguirre A, Gomez Alonso C, Virgos Soriano M.J, Díaz López B, Cannata Andia J. B. Epidemiología de la fractura de cadera en Asturias. Med Clin (Barc) 1995; 105: 281-285.

7. Fernández Portal L, Hernández Morales J, González Orus A, De No Estella L, Martín Cuadrado F. Epidemiología de las fracturas de la extremidad proximal del fémur en la provincia de Salamanca. Revista Ortop. Traum 1992; 3: 329-333.

8. Olmos J.M, Martínez J, García J, Matorras P, Moreno J.J, González Macias J. Incidencia de la fractura de cadera en Cantabria. Med Clín (Barc) 1992; 99: 729-731.

9. Candau E, De la Fuente B, Pozo A, Alvarez J.I, Nieto $C$. Epideminhogía de las fracturas de cadera en la provincia de Valladolid en 1991. Rev Esp Enf Metab Oseas 1993; 2: 73-74

10. Sosa Henriquez M, Segarra Sánchez M.C, Limiriana Canal J.M, Priego López M, Betancor León P. Fractura osteoporótica de la extremidad proximal del fémur. Relación con el hábitat y diferencias hospitalarias. Rev Esp Enf Metab Oseas 1993; 2: 114-117.

11. González Domínguez J, Martínez Concha D, Ca-

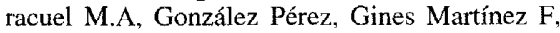
Gala M, Quesada J Manuel. Estudio epidemiológico de las fracturas de cadera de la provincia de Córdoba. Rev Esp Reumatol 1995; 22: 10-14.

12. Rey L, Torrijos A, Armenteros J, Espinosa A, Munuera L, Gijón J. Fracturas de cadera en 1992 en el Área V (Madrid). Rev Esp Reumatol 1995; 22: $39-42$

13. Diez A, Puig J, Martínez M.T, Diez J.L, Aubia J, Vivancos J. Epidemiology of fractures of the proximal fémur associated with osteoporosis in Barcelona, Spain. Calcif Tissue Int 1989; 44: 382-386.

14. Bagur A. Mautalen C, Rubin Z. Epidemiology of hip fractures in an urban population of central Argentina. Osteopor Int 1994; 6: 332-335. 
15. Norton R. et cols. Hip fracture incidence among older in Auckland: a population-based study. Med J. N Z 1995; 108: 426-428.

16. Jequier V, Burnand B, Vader JR, Paccaaud F. Hip fracture incidence in the canton of Vaud, Switzerland, 1986-1991. Osteopor Int .1995; 5: 191-195.

17. Luthje P, Santavirta S, Nurmi Y, Honkanen R, Heiliovaara M. Increasing incidence of hip fractures in Finland. Arch Ortop Trauma Surg 1993; 112: $280-282$.

18. Oriente P, Del Puente A, Scarpa R, Mantova D, Mandes M.G, Vuoso U. The extremely low incidence of proximal femoral fractures due to osteoporosis in the population on the island of Isquia. Ann Ital Med Int. 1995; 10: 159-162.

19. Lustenberger A. Epidemiology of trochanteric femoral fractures over 2 decades (1972-1988). Unfallchirurg. 1995; 98: 278-282.

20. Diez Pérez A. et al. Aproximación a los costes de la fractura osteoporótica de fémur en España. Med Clín (Barc) 1989; 92: 721-723.

21. Madhok R, Melton LJ, Atkinson EJ, O'Fallon WM, Lewallen DG.Urban vs rural increase of hip fracture incidence. Age and sex of 901 cases 1980-89 in Olmsted Country. USA. Acta Orthop Scand 1993; 64: 543-548.

22. Codine O, Braun V, Dhoms G. Facteurs conditionannatt de la reprise de la marche apres fracture du col femoral chez les personnes agees. Ann Readaptation Med Phys 1995; 38: 391

23. Neumann J., Morscher E. Elective total hip prothesis management in patients over 80 years of age. Orthopeda 1994; 23:50-59.
24. Borgquist L, Thongren KG. The financial cost of hip fractures. Acta Orthop Belg 1994; 60: 102105.

25. Berglund-Roden $M$, Swierstra $B$, Winstrand $H$, Thorngren $\mathrm{Kg}$. Prospective comparison of hip fracture treatment. 856 cases folloged for 4 months in the Netherlands and Sweden. Acta Orthop Scand 1994; 65: 287-294.

26. Stone SP, Ali B, Auberleek I, Thopsell A, Young A. The Barthel index in clinical practice: use on a rehabilitation ward for elderly people. J R Coll Physicians Lond. 1994; 28; 419-423.

27. Hernández García M.A, Santamaría Pastor M, Rodríguez Salvasen F.J. La estancia hospitalaria en los pacientes traumatológicos y su relación con la rehabilitación y la problemática social. Rehabilitación (Mar) 1994; 22 Supl 4: 227-231.

28. Flanagan SR, Ragnarsson KT, Ross MK, Wong DK. Rehabilitation of geriatric orthopaedic patient. Clin Orthop 1995; 316: 80-92.

29. Birge SJ, Morrow-Howell N, Proctor EK. Hip fracture. Clin Geriatr Med 1994; 10: 589-609.

30. Weatherall M. One year follow up of patients with fracture of the proximal fémur. N Z Med J 1994; 107(983): 308-9.

31. Guerrero Fernández M, Gari Parera J. Definición cualitativa y cuantitativa de la patología de cadera en un Hospital de agudos. Mapfre Med 1993; 4 : $85-94$.

32. González Macías J. El sol, la vitamina D, la osteoporosis y la cara oculta de la luna. Med Clín (Barc) $1996 ; 2: 60-62$ 